\title{
Long history of IAM comparisons
}

To the Editor - We agree with the point made in a recent Editorial in this journal ${ }^{1}$ that the assumptions behind models of all types, including integrated assessment models (IAMs), should be as transparent as possible. However, it is incorrect to imply that the IAM community is just "now emulating the efforts of climate researchers by instigating their own model intercomparison projects."

In fact, model comparisons for integrated assessment and climate models followed a remarkably similar trajectory. Early general circulation model (GCM) comparison efforts ${ }^{2}$ evolved to the first Atmospheric Model Inter-comparison Project (AMIP), which was initiated in the early $1990 \mathrm{~s}^{3}$. Atmospheric models developed into coupled atmosphere-ocean models (AOGCMs) and results from the first Coupled Model Inter-Comparison Project (CMIP1) became available about a decade later ${ }^{4}$.

Results of first energy model comparison exercise, conducted under the auspices of the Stanford Energy Modeling Forum, were published in $1977^{5}$. A summary of the first comparison focused on climate change was published in $1993^{6}$. As energy models were coupled to simple economic and climate models to form IAMs, the first comparison exercise for IAMs (EMF 14; https://emf.stanford.edu/projects) was initiated in 1994, and IAM comparison exercises have been ongoing since this time $\mathrm{e}^{7-10}$ — and were recently assessed in the latest IPCC report $^{11}$ — including a publicly accessible database of scenarios (https://secure.iiasa.ac.at/web-apps/ ene/AR5DB).

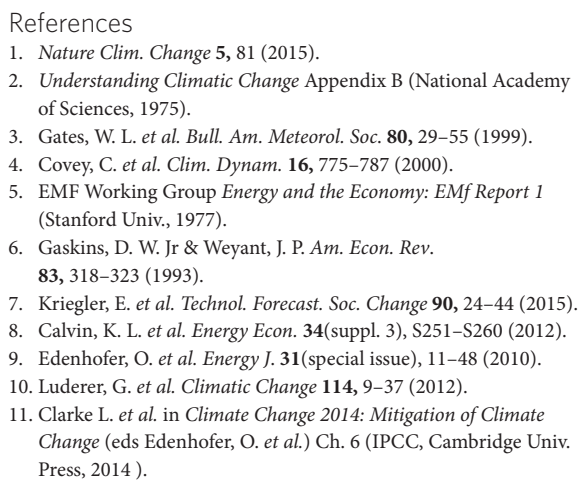

Steven J. Smith ${ }^{1 \star}$, Leon E. Clarke',

James A Edmonds', Jiang Kejun²,
Elmar Kriegler ${ }^{3}$, Toshihiko Masui ${ }^{4}$, Keywan Riahi ${ }^{5}$, Priyadarshi R. Shukla ${ }^{6}$, Massimo Tavoni ${ }^{7}$, Detlef P. van Vuuren ${ }^{8,9}$ and John P. Weyant ${ }^{10}$

1Joint Global Change Research Institute, Pacific Northwest National Laboratory, College Park, Maryland 20740, USA. 'Energy Research Institute, B1505, Guohong Building, Jia.No.11, Muxidibeili, Xicheng District, Beijing 100038, China. ${ }^{3}$ Potsdam Institute for

$\square$ Climate Impact Research, PO Box 601203, 14412 Potsdam, Germany. ${ }^{4}$ National Institute for Environmental Studies, 16-2 Onogawa, Tsukuba, Ibaraki 305-8506, Japan. ${ }^{5}$ International Institute for Applied Systems Analysis (IIASA), Schlossplatz 1, A-2361 Laxenburg, Austria. ${ }^{6}$ Indian Institute of Management, Vastapur, Ahmedabad 380015 India. ${ }^{7}$ Fondazione Eni Enrico Mattei (FEEM), Corso Magenta 63, 20123 Milano, Italy. ${ }^{8} \mathrm{PBL}$ Netherlands Environmental Assessment Agency, PO Box 303, 3720 AH Bilthoven, the Netherlands. ${ }^{9}$ Faculty of Geosciences, Utrecht University, 3584 CS Utrecht, the Netherlands. ${ }^{10}$ Department of Management Science and Engineering, Stanford University, Stanford, California 94305, USA. *e-mail: ssmith@pnl.gov

\section{CORRESPONDENCE:}

\section{Strategies for changing the intellectual climate}

To the Editor - Castree et al. ${ }^{1}$ are correct that a 'single, seamless concept of integrated knowledge' cannot do justice to the diversity of meanings that need to be brought to bear in addressing the challenges of global environmental change. We also agree with them that environmental social sciences and humanities (ESSH) can make important contributions to global environmental change (GEC) science. However, their charge that we ignore the full range of anthropological contributions to understanding of climate change reflects a misreading of our recent Perspective ${ }^{2}$ in this journal, as we only attempted to discuss a few exemplary strands of the many contributions from anthropology to a richer understanding of climate change (for a more detailed discussion, see our forthcoming edited volume ${ }^{3}$ ).

Secondly, Castree et al. suggest that we are reinforcing the status quo in GEC science and 'pulling our punches' by using terms common in Earth systems science (such as system and mechanism). Our use of such terms reflected a strategy to use familiar language to raise awareness of anthropological contributions little known to most GEC scientists, along the lines of the 'clumsy solutions' proposed by anthropologist Steven Rayner ${ }^{4}$. Rayner calls for these solutions to 'wicked problems' such as climate change - problems marked by deep underlying conflicts about the nature of the problem itself - because they can allow different actors to work together without sharing ethical or epistemological principles. We agree with Castree et al. that other strategies are possible, but not that theirs is the only route to a wider dialogue.

Castree et al. focus on three texts to illustrate how GEC scientists evoke the notion of seamless, totalizing knowledge. They single out the use of terms such as 'integration' in discussions of knowledge to 